\title{
Leakage Point Locating Algorithm Based on Generalized Mutual Correlation in Underground Water Pipeline
}

\section{Zexing Chen ${ }^{1}$}

School of Mathematics and Computer Science, Wuhan Polytechnic University

Wuhan,430023, China

E-mail:1564233259@qq. com

Xiaoqing Zhang ${ }^{23}$

School of Mathematics and Computer Science, Wuhan Polytechnic University

Wuhan,430023, China

E-mail:51449902@qq. com

\section{Xiutian Wang ${ }^{\mathrm{a}}$, Renfeng Liu ${ }^{\mathrm{b}}$, Aodong Xue}

School of Mathematics and Computer Science, Wuhan Polytechnic University

Wuhan 430023, China

E-mail: ${ }^{a} 2660409665 @ q q$. com; ${ }^{b} 9231670 @ q q$. com; ${ }^{c} 132605794720163$. com

\begin{abstract}
In order to solve the problem of leakage point positioning in the underground water pipeline network, a generalized cross-correlation algorithm GCC based on fast Fourier transform is proposed in this paper. By suppressing the disturbance of the noise signal to time delay estimation, our algorithm can effectively reduce the error rate, improve the operation speed and make a signal source processing based on dynamic signal capturing to find the time delay points more precisely. Besides, the error can be reduced to a minimum range by our generalized crosscorrelation algorithm so that the precise positioning on the underground water pipeline network can be realized. The simulation experimental results show that our generalized cross-correlation algorithm GCC is effective, feasible and applicable to detect and localize the leakage point in the undergound water pipeline.
\end{abstract}

CENet 2017

22-23 July 2017

Shanghai, China

\footnotetext{
${ }^{1}$ Speaker

${ }^{2}$ Corresponding Author

${ }^{3}$ This study is supported by Youth Scientific Research Project of Education Department in Hubei Province (Q20171708)
} 


\section{Introduction}

The time delay estimation is a common method for the pipeline leakage detection, which can calculate the location of leakage points by analyzing the time delay of the test point signal; however, there are unpredictable noises in the detection of actual environment, which has seriously affected the accuracy of the time delay estimation[1]. In order to improve the detection accuracy and overcome the influence of the noise signal, the numerical optimizaion algorithm is needed to further derive the objective function [2].

The time delay estimation is of great application value in the target positioning [3]. In order to locate the position of the leakage, the cross-correlation of the filtered leakage acoustic signal can calculate the time delay [4]. And, a design adopted the correlation algorithm was also introduced in respect of how to locate the water leakage of underground pipeline [5]. The realtime collection, filting and correclating of two channel audio signal are evaluated in the experiment. However, the variety of time delay is different from performance while it needs to consider the interference from practicality and real-time in the noisy environment. The correlation method is the general method of time delay estimation according to the principle of calculating the time delay estimation based on the correlation of signal [6]. The principle of this method was simple and its computation was small as a kind of basic algorithm of time delay estimation [7]. But in the practical application, the noise actually is multi-faceted and biological. In this case, the correlation estimation delay appears powerless.

GCC can transform the Fourier fastly and the reciprocal power weighted computation of the signal based on the interrelated algorithm. GCC is advantageous to the data in pre-filter processing, which strengthens the spectral component of the source signal in reception and improves SNR[8]. The acoustic signal in the leaky test is a periodic sine wave weighted by PHPA to obtain higher time-delay precision.

\section{Leakage Point Detecting Mode}

\subsection{Two-point Detection Principle}

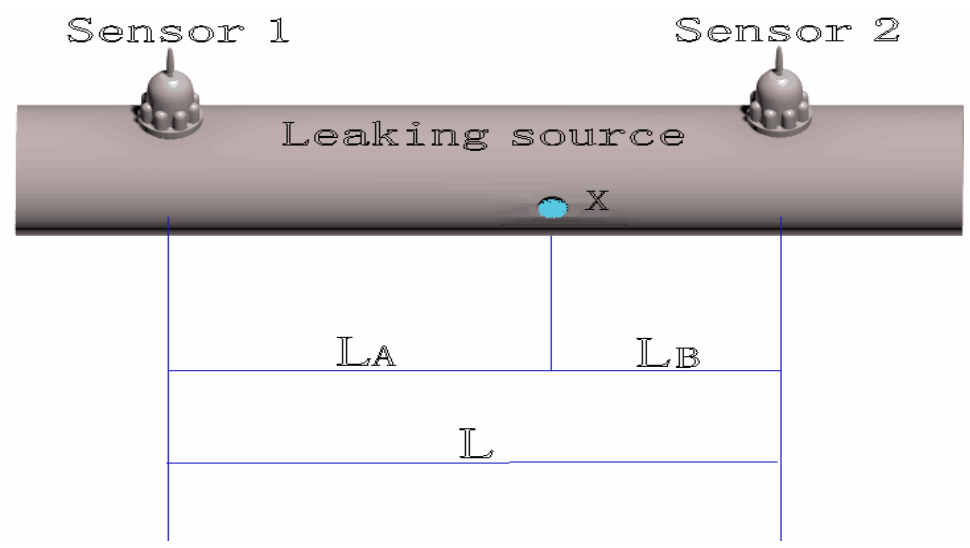

Figure 1: Schematic Diagram of Two-point Detecting principle

As showed in Figure 1, let the transmission time from the signal source of leakage point $\mathrm{X}$ to the sensor 1 be $t$, the propagation speed of the acoustic signal on the pipeline be $V$. Then, the distance between the leakage point and the sensor 1 is $L_{A}$ and

$$
L_{A}=V \times t
$$


$\Delta t$ denotes the data time delay obtained by two sensors. Obviously, the distance between theleakage point and the Sensor 2 is $L_{B}$ and

$$
L_{B}=V \times(t+\Delta t)
$$

The distance between the two sensors is $L$ and

$$
L=L_{A}+L_{B}
$$

Assuming the sensor collects $N$ signal points per second, let the delay points of the data obtained by two sensors be $n$. Then, the delay $\Delta t$ is

$$
\Delta t=n / N
$$

In summary, we can get $L_{A}$,

$$
L_{A}=\frac{L-V \times n / N}{2}
$$

\section{Generalized Cross-correlation Algorithm for Delay}

\subsection{Principle of Generalized Cross-correlation Algorithm}

In the detection of urban pipeline leakage, the time delay of detecting two signals can be obtained by detecting the cross-correlation function of the two signals[9]. However, in actual application, the spectral characteristics of the signal and noise cannot be predicted accurately but be only predictted by the sampling of time. The peak of the cross-correlation function will be affected when the signal spectrum feature is not ideal or the SNR is relative low. In order to reduce the noise influence on the cross-correlation time delay and improve the accuracy of time delay, two cross-power density functions of receiving signals needed to be processed by weighted filtering, called as the generalized cross-correlation algorithm (GCC).

The method of generalized correlation time delay estimation is to suppress noise power and obtain more ideal time delay. The generalized correlation time delay estimation variance of the weighted function theory can be theoretically limited. Assuming that the signal that the leaking point sends is $S(n)$, the signal Sensor 1 receives is $x_{1}(n)$ and the signal Sensor 2 receives is $x_{2}(n)$, we can have

$$
X_{1}(n)=S\left(n-\frac{L_{A}}{V T}\right)+n_{1}(n) \quad, \quad X_{2}(n)=\alpha S\left(n-\frac{L_{B}}{V T}\right)+n_{2}(n)
$$

where $n_{1}(n)$ and $n_{2}(n)$ are the noise signals, $T$ is the sampling period and $\alpha$ is the attenuation factor.

The generalized cross-correlation function of $x_{1}(n)$ and $x_{2}(n)$ is:

$$
R_{X_{1} X_{2}}(r)=E\left[x_{1}(n-r) x_{2}(n)\right]=\alpha R_{n}\left(r-\frac{L_{B}}{V T}+\frac{L_{A}}{V T}\right)+\alpha R_{n_{1} S}\left(r-\frac{L_{B}}{V T}\right)+R_{m_{1}}\left(r+\frac{L_{A}}{V T}\right)+R_{n_{1} n_{2}}(r)
$$

The generalized cross-correlation method cannot detect the peak of Formula (3.2), but do the weighted filter processing for $R_{X_{X X}}(r)$ filter to get the generalized cross-correlation function.

$$
R_{y_{1} y_{2}}(r)=\xi^{-1} G_{y_{1} y_{2}}(w)=\xi^{-1}\left[H(w) G_{x_{1} x_{2}}(w)\right]
$$

$G_{y 122}(w)$ is the spectral density function of generalized cross-correlation, $H(w)=H_{2}(w) H_{1}(w)$ is the related weighted function. The time delay estimation can be obtained when detecting the peak of Formula (3.3). When $r=\left(L_{A}-L_{B}\right) / v t, R_{y 1 y 2}(r)$ reaches the maximal and the signals $x_{1}(n)$ and $x_{2}(n)$ have the greatest relevance. With $\Delta t=\left(L_{A}-L_{B}\right) / v$, substitute $r$ into it, thus we can have

$$
\Delta t=r \times t
$$

\subsection{Estimation of Generalized Cross-correlation Function}


The time delay estimation needs to be realized by using different weighted functions. By comparing the maximum likelihood weighted ML or HT,

$$
H(w)=\frac{\left|\gamma_{12(w)}\right|^{2}}{\left|G_{x_{1} x_{2}}(w)\right|\left[1-\left|\gamma_{12}(w)\right|^{2}\right]},\left|r_{12(w)}\right|^{2}=\frac{\left|G_{x_{1} x_{2}}(w)\right|^{2}}{G_{x_{1} x_{2}}(w) G_{x_{2} x_{2}}(w)} \text { is weighted by SNR }
$$

square in the small signal-to-noise ratio, and weighted by SNR in the large signal-to-noise ratio, which is the optimal estimation method for estimating the variance of time delay. The generalized mutual spectral density with maximal likelihood weighted is:

$$
G_{y_{1} y_{2}}(w)=\frac{\left|\gamma_{12(w)}\right|^{2}}{\left|G_{X_{1} X_{2}}(w)\right|\left[1-\left|\gamma_{12}(w)\right|^{2}\right]} G_{x_{1} x_{2}}(w)
$$

The parameter includes: $G_{X_{1 X} X_{1}}(w), G_{x 1 x_{2}}(w)$ and $G_{x 2 x_{2}}(w) . G_{X_{1} X_{1}}(w)$ and $G_{x 2 x_{2}}(w)$ are the power spectral density functions of the signals $x_{1}(n)$ and $x_{2}(n)$. The estimation of power spectral density can be used in the cycle graph method,

$$
\hat{G_{X X}}\left(w_{k}\right)=\frac{1}{M}\left|X(k)^{2}\right|(k=0,1,2, \ldots, M-1)
$$

$G_{x \mid x_{2}}(w)$ is the mutual spectral density function of the signals $x_{1}(n)$ and $x_{2}(n)$. In the generalized stationary random process of state experience, the estimation is calculated:

$$
\hat{R_{12}}(m)=\frac{1}{M} \sum_{n=0}^{N-1} X_{1}(n-m) X_{2}(n)=\frac{1}{M} X_{1}(-n) \times X_{2}(n)
$$

Formula (3.7) can be expressed in the frequency domain as:

$$
\hat{G_{12}}\left(w_{k}\right)=\frac{1}{M} X_{x_{1}}^{n}(k) X_{x_{2}}(k)(k=0,1,2, \ldots, N-1)
$$

The Fourier series transform can be used to calculate the solution of Formula (3.8) quickly[3].

\subsection{Flow of Generalized Cross-correlation Algorithm}

(1) The acoustic wave signals saved by Sensor 1 and Sensor 2 in the same time are defined as the vector $a[n]$ and $b[n]$.

(2) Select continuously the signal save point of $x$ in location a as the basic comparison metric.

(3) Obtain the delay point $n$ according to the cross-correlation algorithm.

(4) Substitute into the equation of computing $L_{A}$ and get the distance between the leaked point and two sensors.

(5) Repeat Steps 2) 4) until satisfying the required precision.

$F S$ denotes the sampling frequency and $n$ denotes the amount of sampling points. Using the fast Fourier transform on $A$ and $B$ signals, then

$$
X_{1}=F F T(A, 2 \times N), X_{2}=F F T(B, 2 \times N)
$$

Then we need to solve the mutual power function $P_{X Y}$, which denotes the energy correlation of $A$ and $B$ signals of the same frequency. In the time domain representation, $P_{X Y}$ is the conjugate multiplication of the time domain function in $A$ segment and the time domain function of $B$ segment, which can by defined as

$$
P_{X Y}=X_{1} \times \operatorname{conj}\left(X_{2}\right)
$$

The $P_{X Y}$ function presents the time domain correlation of $A$ and $B$, while $P_{X Y}$ can be transferred into $R_{x y}$ by using the inverse Fourier transform, which can be defined as 


$$
R_{x y}=f f t s h i f t\left(\operatorname{real}\left(\text { ifft }\left(P_{X Y}, 2 \times N\right)\right)\right)
$$

The extremum $R_{x y}(n)$ of the function $R_{x y}$ can be explained as: there is the maximal correlation between two information segment $A$ and $B$ when the delay $d=n-N-1$. In other words, the delay point number is $n$ when the acoustic wave signal arrives at the two sensors.

\section{Experimental Analysis}

\subsection{Simulation Environment}

MATLAB 2015a

\subsection{Noise Analysis}

(1) Sonic attenuation

The attenuation of the signal mainly is affected by the propagation distance, the medium of propagation and the absorption of the surrounding medium.

(2) Ambient noise

The ambient noise mainly includes the crash of water flowing (main continue noise sources) and other sudden noises.

\subsection{Actual Requirements of Leaking Point Detection}

In our pipeline leakage location algorithm, the data collection module adopts the time synchronization technology, which must ensure that the synchronization error is less than one milliseconds. The pipeline signal data processing technology can ensure the error between the measured leakage point of pipeline and actual leakage point is less than 1 meter and the rate of the pipeline leakage detection is more than $90 \%$. The system requires no less than 5000 data collecting nodes.

\subsection{Data Analysis}

Two sensors are located on both ends of the pipeline and the data are collected from sensors. We can obtain the waveform in Figure 2. The original acoustic signal collected by two sensors are $\mathrm{S} 1$ and $\mathrm{S} 2$ at the same time. By the normalized processing, we can get the size of 0 point near the waveform and the final is to calculate the delay points $n$ ' through reciprocal function.

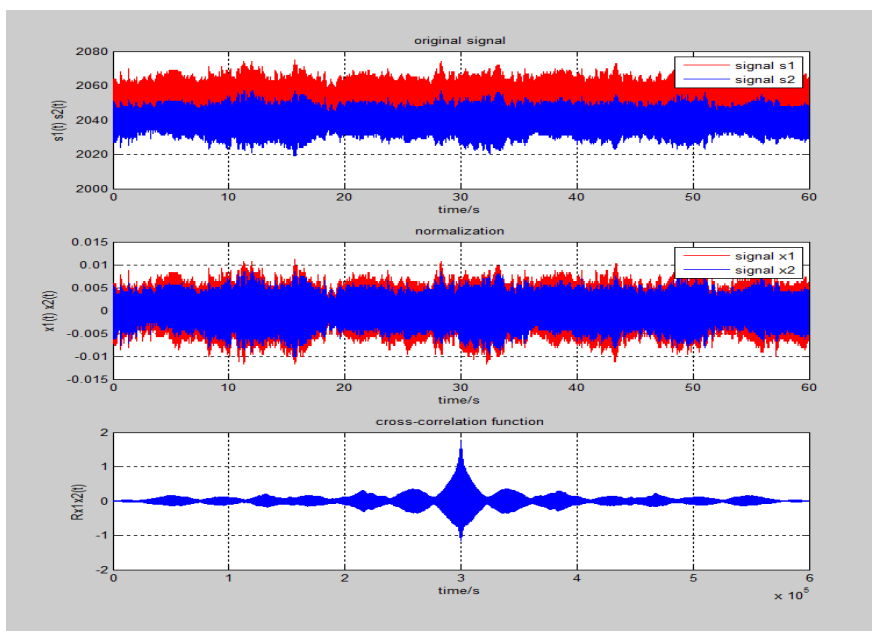

Figure 2: Data Simulation Results 


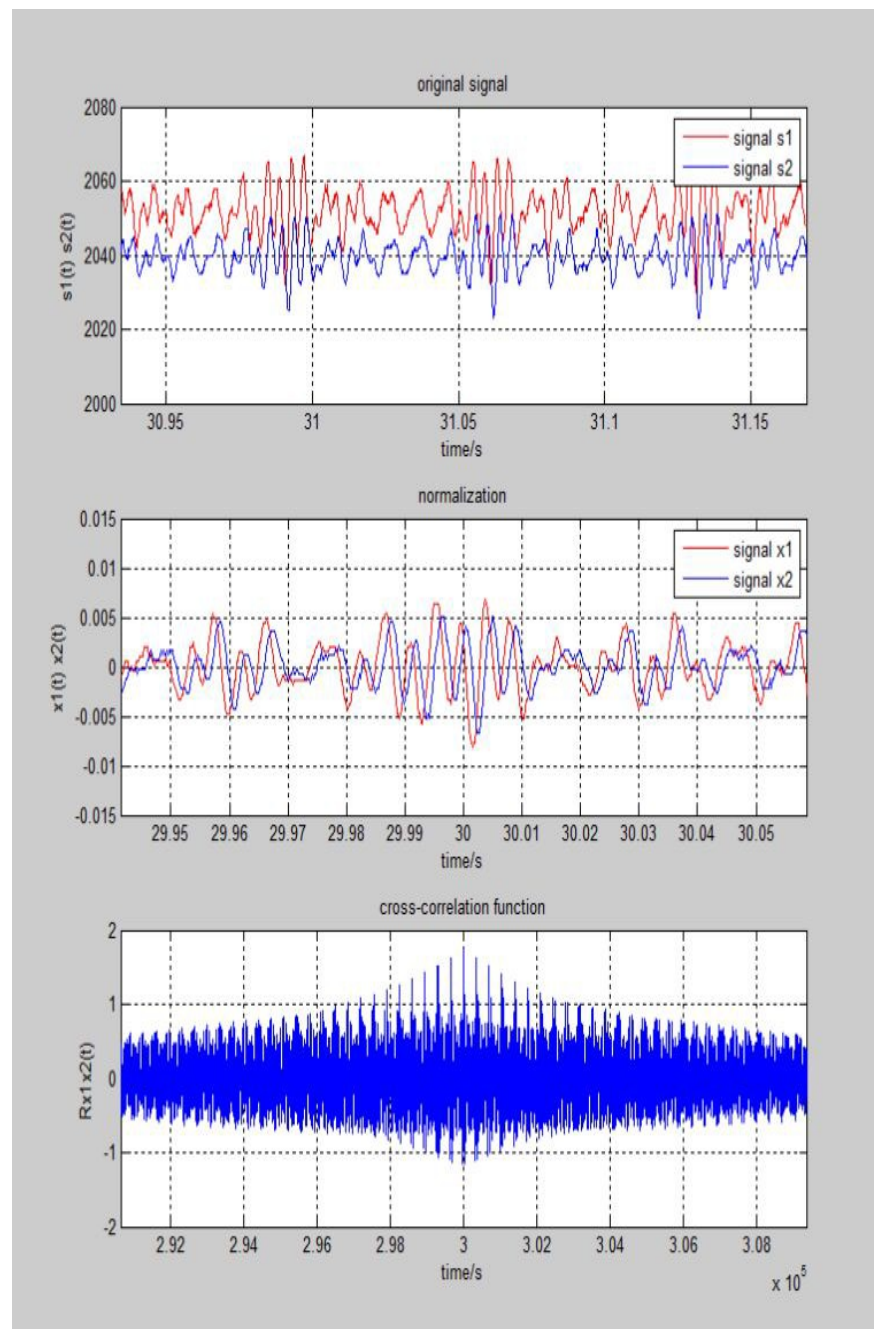

Figure 3: Low-frequency Data Results

\subsection{Detection Example and Error Analysis}

The distance between two sensors is $L$. The speed of the water's sound travel in the pipeline is $1000 \mathrm{~m} / \mathrm{s}$. The sensors collects 5000 data points per second. According to the error analysis in Table 1, the delay points are selected in the range of plus or minus 10. For example:

$$
n=5, \Delta t=5 / 5000=1 / 1000
$$

In summary, we can calculate the distance between the leakage point and Sensor 1,

$$
L_{A}=\frac{L-V \times \Delta t}{2}=\frac{L-\frac{1000 \times 1}{1000}}{2}
$$

In Figure 3, it can be seen that the floating range of the time delay estimation is smaller in line with the error range. In other words, GCC can address the precise positioning problem encountered in most algorithms and improve the detection rate of leakage in pipeline network. At the same time, our algorithm GCC features better stability. 


\begin{tabular}{|l|l|l|}
\hline Delay Points & Distance from Sensor 1 & GCC's Delay Points \\
\hline-5 & 499.5 & -4 \\
\hline 0 & 500 & 0 \\
\hline-9 & 499.5 & -9 \\
\hline-1 & 500 & -1 \\
\hline 10 & 500.5 & 11 \\
\hline-7 & 499.5 & -5 \\
\hline 7 & 499.5 & 6 \\
\hline-1 & 500 & -1 \\
\hline 0 & 500 & 0 \\
\hline 5 & 499.5 & 4 \\
\hline
\end{tabular}

Table 1: Error Table

\section{Conclusion}

The generalized cross-correlation algorithm based on fast Fourier transform can effectively suppress the disturbance of noise signal to time delay estimation and reduce the error rate and improve the operation speed. From the collection of the original signal to the filtering of the noise signals, we make the peak detection to get delay points through cross-correlation of the weighted process function. Our algorithm can increase the length of the Fourier transform and improve the quality of the generalized cross-correlation function, which can improve the time delay estimation by truncating the waveform. The simulation experiments results show that our algorithm is feasible, reliable and capable of implementing the leak detection demand to the actual environment and promoting a level in the mutual correlation algorithm.

\section{References}

[1]Wang J, Liu J, Liu H, et al. Modeling and locating underground water pipe leak with microseismic data $[\mathrm{J}]$. Journal of Applied Geophysics, 136(1):1-8(2017)

[2]Yong Tie, Yang Liu, Shuhua Li. Application of generalized correlated time delay estimation method to leak detection[D]. Volume II of March 2007 38th(In Chinese)

[3] Li W,Guo M,Li Z,Study on the adaptive time delay estimation method for leakage locating of the water supply pipeline[J].Process Autonmation Instrumentation,36(8):10-14(2015)(In Chinese)

[4]W Shanshan,H Guobing, G Bing, et al.Variational model decomposition(VMD) based method of leakage locating in water delivery lines[J].Sensor Letters,14(8):846-851(2016)

[5]Ma Y,Li S.The application of correlation algorithm in the location of underground pipeline water [C].In Conference on Future Computer and Communication.IEEE,481-484(2010)

[6]Chen L,Yu G,Song Y,et al.Precision analysis based on acoustic positioning algorthm[J].Science of Surveying and Mapping,41(5):21-25(2016)(In Chinese)

[7]Lifang Li,Qinghua Liu. Study on time delay estimation of impulse noise[J].Electro-acoustic Technology.32(8):121-125(2008)(In Chinese)

[8]Lingli Chen, Jie Li. Study on leakage analysis of water supply pipe network[J].Seismic Engineering and Engineering Vibration,(23)4:115-121(2003)(In Chinese)

[9]Arifianto D, Wirawan, Atmaja T, et al. Azimuth Tracking of Underwater Moving Sound Source Based on Time Delay Estimation Using Hydrophone Array[J]. Procedia Engineering,170(1):169176(2017) 